\title{
RATIONALITY OF REPRESENTATIONS OF LINEAR LIE GROUPS
}

\author{
DONG HOON LEE AND TA-SUN WU
}

(Communicated by Jonathan M. Rosenberg)

\begin{abstract}
We are concerned with real linear Lie groups $G$ having the property that every finite-dimensional continuous representation of $G$ is rational.
\end{abstract}

By a rational representation of a real linear Lie group $G \subset \mathrm{GL}(n, \mathbb{R})$ we mean a continuous representation of $G$, which is the restriction to $G$ of a rational representation $\rho$ of an algebraic subgroup of $\operatorname{GL}(n, \mathbb{R})$ containing $G$. If every finite-dimensional continuous representation of $G$ is rational, we say that $G$ has the property $(\mathrm{R})$. The importance of Lie groups with this property derives, for example, from the result of Sugiura [6, Theorem 2], which states that the duality theorem holds for a Lie group $G$ if and only if $G$ is the underlying Lie group of an algebraic group and every continuous representation of $G$ is rational. Sugiura also proved that the underlying Lie group of a real semisimple algebraic group has the property $(\mathbf{R})$ [7, Theorem 3]. However, this result seems to have flaws in its proof, and there is indeed a counterexample, which we present at the end of the paper. In this paper, we give several properties for real linear Lie groups, each of which is equivalent to the property (R) (Theorem 1), and then we study conditions under which the underlying Lie group of a real algebraic groups (or more generally a prealgebraic group) has the property (R) (Theorems 2-4).

Notation and convention. Throughout this work, a Lie group always refers to a real Lie group unless some qualification is made explicitly, and a representation of a Lie group is always assumed to be finite-dimensional and over $\mathbb{C}$ (i.e., homomorphism into $\mathrm{GL}(n, \mathbb{C})$ for some $n$ ). A real (resp. complex) algebraic group is a subgroup of $\mathrm{GL}(n, \mathbb{R})(\operatorname{resp} . \mathrm{GL}(n, \mathbb{C}))$ that consists of all invertible matrices whose coefficients annihilate some set of polynomials with real (resp. complex) coefficients, in $n^{2}$ indeterminates. By a prealgebraic group, we mean a (topologically) open subgroup of a real algebraic group of finite index. The class of prealgebraic groups include connected semisimple linear Lie groups and also the (topological) identity component of any real algebraic group. In the actual application of algebraic group theory to the study of Lie groups, we often find it convenient to deal with prealgebraic groups rather than algebraic groups themselves. The following notation is standard throughout: Given a Lie

Received by the editors May 10, 1990 and, in revised forms, June 26, 1990 and October 1, 1990. 1980 Mathematics Subject Classification (1985 Revision). Primary 22E15, 20 G20. 
group $G, G^{0}$ denotes the identity component of $G$, and for any linear group $G \subseteq \mathrm{GL}(n, \mathbb{C}), G^{*}$ stands for the Zariski closure of $G$ in $\operatorname{GL}(n, \mathbb{C})$.

Given a real linear Lie group $G \subset \mathrm{GL}(n, \mathbb{R})$, the Lie algebra $\mathfrak{G}$ of $G$ is a real subalgebra of the general linear Lie algebra $\operatorname{gl}(n, \mathbb{C})$. Let $\mathfrak{G}_{\mathbb{C}}$ denote the complex subalgebra of $\operatorname{gl}(n, \mathbb{C})$ that is spanned by $\mathfrak{G}$, and let $G_{\mathbb{C}}^{0}$ denote the corresponding analytic subgroup of $\operatorname{GL}(n, \mathbb{C})$. For $x \in G$ and $u \in \mathfrak{G}$, we have $\operatorname{Ad}(x)(u)=x u x^{-1}$, where Ad denotes the adjoint representation of $G$, and hence conjugation by $x$ maps $\mathfrak{G}_{\mathbb{C}}$ into itself. This shows that $G$ normalizes $G_{\mathbb{C}}^{0}$, and the subgroup $G_{\mathbb{C}}=G_{\mathbb{C}}^{0} \cdot G$ of $\operatorname{GL}(n, \mathbb{C})$ becomes a complex Lie group. Note that $G_{\mathbb{C}}^{0}$ is easily seen to be the connected component of $G_{\mathbb{C}}$ containing 1. We call $G_{\mathbb{C}}$ the complexification of the real linear group $G$. Let $G$ be any linear Lie group in $\mathrm{GL}(n, \mathbb{R})$. By a complexification of a representation $\varphi$ of $G$, we mean a complex representation $\varphi^{\prime}$ of $G_{\mathbb{C}}$ such that $\varphi^{\prime}=\varphi$ on $G$. Such a complexification is unique if it exists. Let $G$ be a Lie group with finitely many connected components (such a Lie group is called an f.c.c. group). By the universal complexification of $G$, we mean a pair $\left(G^{+}, j\right)$ where $G^{+}$is a complex Lie group, and $j: G \rightarrow G^{+}$is a continuous homomorphism having the following universal property. Every representation $\rho: G \rightarrow \mathrm{GL}(m, \mathbb{C})$ induces a unique complex representation $\rho^{+}: G^{+} \rightarrow \mathrm{GL}(m, \mathbb{C})$ such that $\rho=\rho^{+} \circ j$, and this property characterizes the universal complexification. The Lie algebra of $G^{+}$is the canonical complex linear extension of the Lie algebra of $G$, and the differential of $\rho^{+}$is the canonical linear extension of the differential of $\rho$. For a detailed discussion, see $[4, \S 4]$. Below we present a brief construction of $\left(G^{+}, j\right)$ for later use. (See the proof of Theorem 1.) Let $S$ denote the simply connected Lie group whose Lie algebra is the complex Lie algebra $\mathbb{C} \otimes \mathfrak{G}$. For any $x \in G$, we consider the complexification $\operatorname{Ad}(x)_{\mathbb{C}}: \mathbb{C} \otimes \mathfrak{G} \rightarrow \mathbb{C} \otimes \mathfrak{G}$ of $\operatorname{Ad}(x): \mathfrak{G} \rightarrow \mathfrak{G}$. There is a unique automorphism $\eta(x)$ of $S$ such that $d \eta(x)=$ $\operatorname{Ad}(x)_{\mathbb{C}}$. Form the semidirect product $H=S \times_{\eta} G$ of $S$ and $G$ relative to the analytic homomorphism $\eta: G \rightarrow \operatorname{Aut}(S)$. Given any representation $\rho: G \rightarrow$ $\mathrm{GL}(m, \mathbb{C})$, there exists a unique representation $\rho^{\prime}: S \rightarrow \mathrm{GL}(m, \mathbb{C})$ such that $d \rho^{\prime}=(d \rho)_{\mathbb{C}}$, where $(d \rho)_{\mathbb{C}}: \mathbb{C} \otimes \mathfrak{G} \rightarrow \mathrm{GL}(m, \mathbb{C})$ is the $\mathbb{C}$-linear extension of the differential $d \rho$. We define $\rho^{\prime \prime}: H \rightarrow \mathrm{GL}(m, \mathbb{C})$ by $\rho^{\prime \prime}(x, y)=\rho^{\prime}(x) \rho(y)$, and put $K=\bigcap_{\rho} \operatorname{Ker}\left(\rho^{\prime \prime}\right)$, where the intersection is taken over all representations $\rho$ of $G$. If $G^{+}=H / K$, and $j: G \rightarrow G^{+}$is the composition of the canonical maps $G \rightarrow H=S \times_{\eta} G \rightarrow H / K$, then the pair $\left(G^{+}, j\right)$ is the desired universal complexification. For any continuous representation $\rho: G \rightarrow \mathrm{GL}(m, \mathbb{C}), \rho^{+}$ is induced by $\rho^{\prime \prime}$.

Given a Lie group $G$ and a continuous representation $\rho: G \rightarrow \operatorname{GL}(n, \mathbb{C})$, let $[\rho]$ denote the $\mathbb{C}$-linear space spanned by the matricial coefficients of the representation $\rho$, and let $R(G)$ denote the union of the linear spaces $[\rho]$, as $\rho$ runs over all continuous representations. $R(G)$ is a $\mathbb{C}$-algebra under the usual sum and product, and functions in $R(G)$ are called representative functions of $G$. Let $R_{\mathbb{R}}(G)$ be the real subalgebra of $R(G)$ consisting of all representative functions $f$ of $G$ with $\bar{f}=f$. Since the real and imaginary parts of a representative function on $G$ belong to $R_{\mathbb{R}}(G)$, it follows that if $R(G)$ is finitely generated as a $\mathbb{C}$-algebra, then $R_{\mathbb{R}}(G)$ is finitely generated as an $\mathbb{R}$-algebra. An easy and direct argument establishes the following:

Lemma 1. Let $\tau: G \rightarrow \mathrm{GL}(m, \mathbb{C})$ be a continuous representation of a Lie group 
$G$ such that the induced representation $\tau^{+}: G^{+} \rightarrow \mathrm{GL}(m, \mathbb{C})$ is faithful. If $S$ is a finite-dimensional subspace of $R(G)$ that contains $[\tau]$ and if $S$ is an invariant under the left translations of elements in $G$, then the representation of $G^{+}$in $S$ that is induced from the representation of $G$ in $S$ via the left translations is faithful.

Given a real linear Lie group $G \subset \mathrm{GL}(n, \mathbb{R})$, let $A(G)$ be the algebra of the functions on $G$ obtained by restriction to $G$ of all polynomial functions on $\mathrm{GL}(n, \mathbb{R})$. We call this algebra the affine algebra of $G$. We note that if $\mu$ denotes the inclusion of $G$ into $\mathrm{GL}(n, \mathbb{R})$, then $A(G)=\mathbb{R}\left[\ldots, \mu_{i j}, \ldots,(\operatorname{det} \circ \mu)^{-1}\right]$, where $\mu_{i j}$ denotes the $(i, j)$-component of $\mu$, and we have $A(G) \subset R_{\mathbb{R}}(G)$. If $G$ is an algebraic group, then $A(G)$ is the affine algebra of $G$ in the usual sense.

Definition [2]. A real linear Lie group $G \subset \mathrm{GL}(n, \mathbb{R})$ is said to be well embedded if every continuous representation of $G$ has a complexification.

Here is our first result.

Theorem 1. Let $G \subset \mathrm{GL}(n, \mathbb{R})$ be an f.c.c. real linear Lie group, and assume that the algebra $R(G)$ of all representative functions of $G$ is finitely generated. The following are equivalent:

(i) $G$ is well embedded.

(ii) The affine algebra of $G$ is $R_{\mathbb{R}}(G)$.

(iii) $G$ has the property $(\mathbf{R})$.

(iv) The inclusion $l$ of $G$ into its Zariski closure $G^{*}$ in $\mathrm{GL}(n, \mathbb{C})$ is the universal complexification of $G$.

Proof. We view $G$ as a subgroup of $\mathrm{GL}(n, \mathbb{C}) . \quad$ (i) $\rightarrow$ (ii). We retain the notation introduced earlier in the construction of the universal complexification. The complexification $G_{\mathrm{C}}^{0}$ of the identity component $G^{0}$ of $G$ is the analytic subgroup of $\mathrm{GL}(n, \mathbb{C})$ whose Lie algebra is the complex Lie subalgebra in $\operatorname{gl}(n, \mathbb{C})$ that is generated over $\mathbb{C}$ by the Lie algebra of $G$. Let $\mu: G \rightarrow \mathrm{GL}(n, \mathbb{C})$ denote the inclusion. We first show that $\mu^{+}$is faithful. Let $S$ denote the simply connected analytic group with its Lie algebra $\mathbb{C} \otimes \mathfrak{G}$, and let $\sigma: S \rightarrow G_{\mathbb{C}}^{0}$ denote the universal covering of $G_{\mathbb{C}}^{0}$. For any continuous representation $\rho: G \rightarrow \mathrm{GL}(m, \mathbb{C})$, let $\rho_{\mathbb{C}}: G_{\mathbb{C}} \rightarrow \mathrm{GL}(m, \mathbb{C})$ denote the complexification of $\rho$. Then the representation $\rho^{\prime}: S \rightarrow \mathrm{GL}(m, \mathbb{C})$ induced by $\rho$ satisfies $\rho^{\prime}(x)=\rho_{\mathbb{C}}(\sigma(x)), x \in S$. Since $\mu_{\mathbb{C}}$ is the inclusion $G_{\mathbb{C}} \subset \mathrm{GL}(n, \mathbb{C})$, $\mu^{\prime}(x)=\mu_{\mathbb{C}}(\sigma(x))=\sigma(x)$ for all $x \in S$ and $\mu^{\prime \prime}: S \rightarrow \mathrm{GL}(m, \mathbb{C})$ is therefore given by $\mu^{\prime \prime}(x, y)=\sigma(x) y$. We also have $\operatorname{Im}\left(\mu^{\prime \prime}\right)=G_{\mathbb{C}}$. We note that $\operatorname{Ker}\left(\mu^{\prime \prime}\right)=\left\{\left(x, \sigma(x)^{-1}\right): x \in \sigma^{-1}\left(G_{\mathbb{C}}^{0} \cap G\right)\right\}$, and for each continuous representation $\rho$ of $G, \rho^{\prime \prime}$ maps $\operatorname{Ker}\left(\mu^{\prime \prime}\right)$ to 1 . Thus we have $\operatorname{Ker}\left(\mu^{\prime \prime}\right) \subset \operatorname{Ker}\left(\rho^{\prime \prime}\right)$ for all continuous representations $\rho$ of $G$, and from the definition of $\mu^{+}$, it is easy to see that $\mu^{+}$is faithful.

Let $\mu^{0}$ denote the direct sum of the representation $\mu$ and its dual. Then the subspace $\left[\mu^{0}\right]$ of the representative functions associated with $\mu^{0}$ is stable under the left translations and the antipodal map $f \rightarrow f^{\prime}$, where $f^{\prime}$ is defined by $f^{\prime}(x)=f\left(x^{-1}\right)$ for $x \in G$. Moreover, $G^{+}$acts on [ $\left[\mu^{0}\right]$ faithfully by the lemma above. Hence by Hochschild and Mostow [4, Theorems 9.4 and 10.1], the algebra $R(G)$ is generated by $\left[\mu^{0}\right]$. Since $A(G)=\mathbb{R}\left[\mu_{11}, \ldots, \mu_{n n},(\operatorname{det} \circ \mu)^{-1}\right]$, 
it follows that $\left[\mu^{0}\right] \cap R_{\mathbb{R}}(G) \subset A(G)$, and hence $R_{\mathbb{R}}(G) \subset A(G)$. Since clearly $A(G) \subset R_{\mathbb{R}}(G)$, it follows that $A(G)=R_{\mathbb{R}}(G)$, proving (ii).

(ii) $\rightarrow$ (iii). Suppose $A(G)=R_{\mathbb{R}}(G)$, and let $\rho: G \rightarrow \mathrm{GL}(m, \mathbb{C})$ be any continuous representation. Let $\bar{G}$ denote the Zariski closure of $G$ in $\operatorname{GL}(n, \mathbb{R})$, and let $y \rightarrow y^{\prime}$ be the canonical isomorphism of $\bar{G}$ with $\operatorname{Hom}_{\mathbb{R}-\mathrm{alg}}(A(G), \mathbb{R})$. Define $\bar{\rho}: \bar{G} \rightarrow \mathrm{GL}(m, \mathbb{C})$ by $(\bar{\rho}(y))_{i j}=y^{\prime}\left(\rho_{i j}\right), y \in \bar{G}$, where $z_{i j}$, for $z \in \mathrm{GL}(m, \mathbb{C})$ denotes the $(i, j)$-entry of $z$, and $\rho_{i j}$ denotes the $(i, j)$ complement of $\rho$. It is clear that $\bar{\rho}$ is a representation of $\bar{G}$, which extends $\rho$, and it remains to show that $\bar{\rho}$ is rational. Since $A(G)=R_{\mathbb{R}}(G)$, and since $\rho_{i j} \in R_{\mathbb{R}}(G)$, we can find an element $P \in A(\mathrm{GL}(n, \mathbb{C}))$ such that $\rho_{i j}=P$ on $G$. Thus $(\bar{\rho}(y))_{i j}=y^{\prime}\left(\rho_{i j}\right)=P(y)$. This shows that $\bar{\rho}$ is a rational map.

(iii) $\rightarrow$ (iv). Let $\rho: G \rightarrow \mathrm{GL}(m, \mathbb{C})$ be any continuous representation of $G$, where $\operatorname{GL}(m, \mathbb{C})$ is viewed as a subgroup of the real algebra group $\operatorname{GL}(2 m, \mathbb{R})$ in the usual way, and let $\bar{G}$ be the Zariski closure of $G$ in $\operatorname{GL}(n, \mathbb{R})$. Since $\rho$ is rational by (iii), $\rho$ can be extended to a rational representation $\bar{\rho}: \bar{G} \rightarrow$ $\mathrm{GL}(2 m, \mathbb{R})$. Let $\bar{\rho}_{\mathbb{C}}: G^{*} \rightarrow \mathrm{GL}(2 m, \mathbb{C})$ denote the complexification of the rational representation $\bar{\rho}$. It is easy to see that $\bar{\rho}_{\mathbb{C}}$ maps $G^{*}$ into $\operatorname{GL}(m, \mathbb{C})$ and that $\bar{\rho}_{\mathbb{C}}$ is the unique extension of $\rho$ to $G^{*}$. This shows that $l: G \rightarrow G^{*}$ is the universal complexification of $G$.

(iv) $\rightarrow(\mathrm{i})$. Let $\rho: G \rightarrow \mathrm{GL}(m, \mathbb{C})$ be any continuous representation. Since the inclusion $l: G \rightarrow G^{*}$ is the universal complexification of $G$, there exists a complex representation $\rho^{*}: G^{*} \rightarrow \mathrm{GL}:(m, \mathbb{C})$, which extends $\rho$. Thus it is enough to show that $G^{*}=G_{\mathbb{C}}$. Indeed, let $\mu: G \rightarrow G(n, \mathbb{C})$ be the inclusion. Thus there exists a unique complex representation $\mu^{*}: G^{*} \rightarrow \operatorname{GL}(n, \mathbb{C})$, which extends $\mu$. By the construction of the universal complexification, we see that $\mu^{*}\left(G^{*}\right)=G_{\mathbb{C}}$. Since $\mu^{*}$ is rational by Hochschild and Mostow [4, Theorem 9.5], $G_{\mathbb{C}}$ is Zariski closed in $\operatorname{GL}(n, \mathbb{C})$, and hence $G^{*} \subset G_{\mathbb{C}}$. The other inclusion is clear, proving that $G^{*}=G_{\mathbb{C}}$.

Remark. The condition that $R(G)$ is finitely generated is essential for the theorem. Indeed, if $G$ is a real algebraic (unipotent) vector group $\mathbb{R}^{n}$ (e.g. the $n$-dimensional linear group consisting all matrices in $\mathrm{GL}(n+1, \mathbb{R})$ of the form

$$
\left[\begin{array}{ccccc}
1 & 0 & \cdots & 0 & t_{1} \\
0 & 1 & 0 & \ddots & t_{2} \\
& 0 & \ddots & 0 & \vdots \\
& & & 1 & t_{n} \\
0 & & \cdots & 0 & 1
\end{array}\right]
$$

with $\left.t_{1}, \ldots, t_{n} \in \mathbb{R}\right)$ then the underlying Lie group of $G$ is clearly well embedded while its affine algebra $\mathbb{R}\left[x_{1}, \ldots, x_{n}\right]$ is not equal to $R_{\mathbb{R}}(G)$.

Remark. Every compact linear group $G$ is well embedded. To see this, let $\mu$ be the inclusion map of $G$ into, say, $\operatorname{GL}(n, \mathbb{R})$. Then we have $A(G)=$ $\mathbb{R}\left[\mu_{11}, \ldots, \mu_{n n},(\operatorname{det} \circ \mu)^{-1}\right]$. On the other hand, $R_{\mathbb{R}}(G)$ is generated by the $\mu_{i j}, 1 \leq i, j \leq n$ by Chevalley [1, Proposition 3, p. 190]. Thus $A(G)=R_{\mathbb{R}}(G)$ and by Theorem 1 above, $G$ is well embedded.

A Lie group $G$ is said to be reductive if there is a faithful representation of $G$ and every continuous representation of $G$ is semisimple. Compact groups and connected semisimple linear Lie groups are all reductive. In general, a 
connected linear Lie group is reductive if and only if the center $Z$ of $G$ is compact and $G / Z$ is semisimple, and in this case $G=Z G^{\prime}$, where $G^{\prime}$ is the commutator subgroup of $G$ that is closed in $G$ (See [3, Chapter XVIII]).

Let $G$ be a linear f.c.c. Lie group, and let $N$ be the analytic subgroup of $G$ whose Lie algebra is the commutator $[\mathfrak{G}, \mathfrak{R}$ ] where $\mathfrak{R}$ is the solvable radical of $\mathfrak{G}$. Then $N$ is simply connected and nilpotent, and $N$ is equal to the representation radical of $G$ (i.e. the intersection of all kernels of semisimple representations of $G$ ). Every continuous representation of $G$ induces a unipotent representation of $N$. In [4] the following is proved: If $R(G)$ is finitely generated as a $\mathbb{C}$-algebra, then $G$ is a semidirect product $G=N \cdot H$, where $H$ is a maximal reductive subgroup of $G$ [4, Theorem 9.1], and conversely, if an f.c.c. Lie group $G$ is a semidirect product $G=N \cdot H$, where $N$ is the representation radical of $G$ and $H$ is a reductive Lie group, then $R(G)$ is finitely generated [4, Theorem 9.2]. In particular, it follows that if $G$ is an f.c.c. linear Lie group, then $R(G)$ is finitely generated if and only if $R\left(G^{0}\right)$ is finitely generated. We also have the following:

Lemma 2. Let $G_{1}$ be an f.c.c. linear Lie group, and let $\varphi: G_{1} \rightarrow G_{2}$ be a surjective morphism. If $R\left(G_{1}\right)$ is finitely generated, then so is $R\left(G_{2}\right)$.

Proof. Let $N_{i}$ denote the representation radical of $G_{i}(i=1,2)$. By [4, Theorem 9.1], $G_{1}$ is a semidirect product $G_{1}=N_{1} \cdot H$, where $H$ is a reductive subgroup of $G_{1}$. If $\mathfrak{G}_{i}$ denotes the Lie algebra of $G_{i}$ and $\mathfrak{R}_{i}$ denotes the radical of $\mathfrak{G}_{i}(i=1,2)$, then the induced Lie algebra morphism $d \varphi: \mathfrak{G}_{1} \rightarrow \mathfrak{G}_{2}$ maps the ideal $\left[\mathfrak{G}_{1}, \mathfrak{R}_{1}\right]$ onto the ideal $\left[\mathfrak{G}_{2}, \mathfrak{R}_{2}\right]$. Since $N_{i}$ is the analytic subgroup of $G_{i}$ corresponding to the ideal $\left[\mathfrak{G}_{i}, \mathfrak{R}_{i}\right]$, it follows that $\varphi\left(N_{1}\right)=$ $N_{2}$. We then have a semidirect product decomposition $G_{2}=N_{2} \cdot \varphi(H)$. Since $\varphi(H)$ is reductive, $R\left(G_{2}\right)$ is finitely generated by [4, Theorem 9.2].

Next we turn to the study of prealgebraic groups whose underlying Lie groups have the property $(R)$. The following result enables us to consider only connected groups.

Theorem 2. Let $G \subset \mathrm{GL}(n, \mathbb{R})$ be a prealgebraic group such that $R(G)$ is finitely generated. Then $G$ has the property $(\mathrm{R})$ if and only if $G^{0}$ has the property $(\mathrm{R})$ and $G \cap G_{\mathbb{C}}^{0}=G^{0}$.

Proof. Suppose that $G^{0}$ has the property $(\mathrm{R})$ and assume $G \cap G_{\mathbb{C}}^{0}=G^{0}$. By Theorem 1, it is enough to show that $G$ is well embedded. Given any representation $\rho: G \rightarrow \mathrm{GL}(m, \mathbb{C})$, let $\rho^{0}$ denote the restriction of $\rho$ to $G^{0}$. By hypothesis, $\rho^{0}$ has a complexification, say $\rho_{\mathbb{C}}^{0}: G_{\mathbb{C}}^{0} \rightarrow \operatorname{GL}(m, \mathbb{C})$. Define $\bar{\rho}: G_{\mathbb{C}}=G \cdot G_{\mathbb{C}}^{0} \rightarrow \mathrm{GL}(m, \mathbb{C})$ by $\bar{\rho}(x y)=\rho(x) \cdot \rho_{\mathbb{C}}^{0}(y)$ for $x \in G$ and $y \in G_{\mathbb{C}}^{0}$. Since $\rho=\rho_{\mathbb{C}}^{0}$ on $G^{0}$ and since $G \cap G_{\mathbb{C}}^{0}=G^{0}, \bar{\rho}$ is well defined, and it is easy to see that $\bar{\rho}$ is the complexification of $\rho$. Hence $G$ is well embedded.

Now assume that $G$ has the property $(\mathrm{R})$, and let $G^{*}\left(\right.$ resp. $\left.\left(G^{0}\right)^{*}\right)$ denote the Zariski closure of $G$ (resp. $\left.G^{0}\right)$ in $\operatorname{GL}(n, \mathbb{C})$. By Theorem 1 , the inclusion $\mu: G \rightarrow G^{*}$ is the universal complexification of $G$. Pick a representation $\rho$ of $G$ such that $\operatorname{Ker}(\rho)=G^{0}$. $\rho$ is extended to a rational representation $\rho^{*}$ of the algebraic group $G^{*}$, and $\operatorname{Ker}\left(\rho^{*}\right) \supset\left(G^{0}\right)^{*}$. For $x \in G \cap\left(G^{0}\right)^{*}$, $1=\rho^{*}(x)=\rho(x)$ and hence $x \in \operatorname{Ker}(\rho)=G^{0}$. Thus $G \cap G_{\mathbb{C}}^{0}=G^{0}$. Next we show that $G^{0}$ is well embedded (i.e. $G^{0}$ has the property (R) by Theorem 
1). Let $\rho: G^{0} \rightarrow \mathrm{GL}(n, \mathbb{C})$ be a representation of $G_{0}$. Canonically identify $\rho$ with a representation of $G^{0}$ in an $n$-dimensional $\mathbb{C}$-linear space $V$, and let $\rho^{\prime}$ denote the representation of $G$ that is induced by $\rho$ in a $\mathbb{C}$-linear space $W$ containing $V$ as a subspace. Since $G$ is well embedded, $\rho^{\prime}$ has a complexification $\rho_{\mathbb{C}}^{\prime}: G_{\mathbb{C}} \rightarrow \mathrm{GL}(W, \mathbb{C})$. Since the subspace $V$ of $W$ is $G_{\mathbb{C}^{-}}^{0}$ invariant, $\left.x \rightarrow \rho_{\mathbb{C}}^{\prime}(x)\right|_{V}$ defines a representation of $G_{\mathbb{C}}^{0}$ in $V$, which is a desired complexification of $\rho$. Thus $G^{0}$ is well embedded.

A subgroup $H$ of an algebraic group (or more generally a prealgebraic group) $G$ is said to be linearly reductive (resp. unipotent) if every rational representation of $G$, when restricted to $H$, is semisimple (resp. unipotent). $G$ contains the maximal normal unipotent subgroup of $G$, which is obviously an algebraic subgroup of $G$. We call this subgroup the unipotent radical of $G$ and denote it by $G_{u}$. Every algebraic group $G$ is a semidirect product $G_{u} \cdot H$, where $H$ is a maximal linearly reductive subgroup of $G$. This result, which is due to Mostow [5], can be easily extended to any prealgebraic group $G$, and in this case, the maximal linearly reductive subgroup $H$ is prealgebraic.

Lemma 3. Let $G \subset \mathrm{GL}(n, \mathbb{R})$ be a connected prealgebraic group, and assume that $R(G)$ is finitely generated. Then $G_{u}$ is the representation radical of the Lie group $G$ and any maximal linearly reductive subgroup of the prealgebraic group $G$ is a maximal reductive subgroup of the linear Lie group $G$.

Proof. Let $H$ be a maximal linearly reductive subgroup of the prealgebraic group $G$ so that $G$ is a semidirect product $G=G_{u} \cdot H$. Necessarily, $H$ is connected and prealgebraic. The Zariski closure $\bar{H}$ of $H$ in $\operatorname{GL}(n, \mathbb{R})$ is also linearly reductive and hence $\bar{H}$ can be written $\bar{H}=A T S$, where $A$ is a split torus, $T$ an anisotropic torus, and $S$ a semisimple subgroup of $\bar{H}$. Since the connected $H$ is of finite index in $\bar{H}$, it follows that $H=A^{0} T S^{0}$. Note that $A^{0}$ is a central subgroup isomorphic with a finite product of copies of the multiplicative group of positive real numbers, and since the center of $T S^{0}$ is compact, $A^{0} \cap\left(T S^{0}\right)=\{1\}$. Consequently, $A^{0}$ is a direct factor of $H$. Since $R(H)$ is finitely generated, $A^{0}$ is trivial and hence $H=T S^{0}$ is reductive as a Lie group. Now let $N$ be the representation radical of $G$. Clearly $N \subset G_{u}$. Suppose $N$ is properly contained in $G_{u}$. The subgroup $N S$ is normal in $G$ and the quotient group $G / N S \simeq V \times T_{1}$, where $V$ is a vector group and $T_{1}$ is a torus. Hence $R(G / N S) \simeq R\left(V \times T_{1}\right)$ is not finitely generated. On the other hand, since $R(G)$ is finitely generated, $R(G / N S)$ is finitely generated by [4, Theorem 9.2]. This obvious contraction shows that $N=G_{u}$ and our proof is complete.

Corollary (to Lemma 3 ). If $G$ is a connected prealgebraic reductive group such that $R(G)$ is finitely generated, then $G$ is a reductive linear Lie group. Conversely if $G$ is a connected linear reductive Lie group, then $G$ is a linearly reductive prealgebraic group and $R(G)$ is finitely generated.

Proof. The first assertion follows directly from Lemma 3, and the second assertion follows since the reductive group $G$ can be written $G=Z G^{\prime}$, where $Z$ is the center of $G$ which is compact and $G^{\prime}$ is a closed semisimple subgroup.

Let $G \subset \mathrm{GL}(n, \mathbb{R})$ be an f.c.c. linear Lie group with the representation radical $N$ and assume that $R(G)$ is finitely generated. Then $G$ is a semidirect 
product $N \cdot H$, where $H$ is a maximal reductive subgroup of $G$. The semidirect product decomposition $G=N \cdot H$ leads to the decomposition $R(G)=$ $R^{H}(G) \cdot R^{N}(G)$ [4, Proposition 2.4]. Here $R^{H}(G)$ is the image of the map $R(H) \rightarrow R(G)$ that is induced by the projection $G=N \cdot H \rightarrow H$, and $R^{N}(G)$ is the image of the map $g \rightarrow g^{+}: R(G)_{N} \rightarrow R(G)$ where $R(G)_{N}$ is the image, by restriction, of $R(G)$ in $R(N)$, and $g^{+}$, for any $g \in R(G)_{N}$, is given by $g^{+}(n h)=g(n)$ for $n \in N$ and $h \in H$. The injection $R(H) \rightarrow R(G)$ defines an isomorphism $R(H) \rightarrow R^{H}(G)$, and we also have $R(G)_{N}=P(N)$, where $P(N)$ denotes the algebra of all polynomial functions on $N$, when $N$ is identified with its image by a faithful unipotent representation (see [4, Proof of Theorem 9.2]).

If $G$ is a real algebraic group, $G$ is a semidirect product $G=G_{u} \cdot H$, where $\mathrm{H}$ is a maximal linearly reductive subgroup of $G$, and this decomposition leads to the decomposition of $A(G): A(G)=A^{H}(G) \cdot A^{N}(G) \simeq A(G)_{N} \otimes A(G)_{H}$, where $A^{L}(G)$ and $A(G)_{L}$ for any subgroup $L$ of $G$ are defined in the same way as $R^{L}(G)$ and $R(G)_{L}$, respectively.

The following result reduces our study of groups with the property $(R)$ to the reductive case.

Theorem 3. Let $G \subset \mathrm{GL}(n, \mathbb{R})$ be a connected prealgebraic group such that $R(G)$ is finitely generated. Then $G$ has the property $(R)$ if and only if its maximal linearly reductive subgroup has the property ( $R)$.

Proof. Let $H$ be a maximal linearly reductive subgroup of $G$. By the decomposition theorem in [5] (adapted to our prealgebraic group), $G$ is a semidirect product $G=G_{u} \cdot H$. By Lemma $3, G_{u}$ is equal to the representation radical $N$ of $G$, and $H$ is a reductive subgroup of the Lie group $G$. Therefore the decomposition $G=G_{u} \cdot H=N \cdot H$ gives decompositions of $A(G)$ and $R(G)$ :

$$
A(G)=A^{H}(G) \cdot A^{N}(G) \simeq A(G)_{N} \otimes A(G)_{H}
$$

and

$$
R(G)=R^{H}(G) \cdot R^{N}(G) \simeq R(G)_{N} \otimes R(H) .
$$

Now assume that $G$ has the property (R), and let $\varphi$ be any continuous representation of $H$. Composing $\varphi$ with the projection $G \rightarrow H$, we have a representation $\varphi^{\prime}$ of $G$, which can be extended to the rational representation $\varphi^{\prime \prime}$ of $G^{*} \subset \operatorname{GL}(n, \mathbb{C}) .\left.\varphi^{\prime \prime}\right|_{H^{*}}$ extends $\varphi$ to $H^{*}$, and this shows that the inclusion $l: H \subset H^{*}$ is a universal complexification of $H$. Thus $H$ has the property $(\mathbf{R})$.

Now assume that $H$ has the property $(\mathrm{R})$. Thus $R(H)=A(H)$, and since $R(G)_{N}=P(N) \quad$ (= the algebra of all polynomial functions on the unipotent group $N)$ (see [4, Proof of Theorem 9.2]), we have $A(H) \otimes P(N)=R(H) \otimes$ $R(G)_{N} \simeq R^{N}(G) \cdot R^{H}(G)$. On the other hand, $A(H) \otimes P(N)$ is clearly mapped into $A(G)$ by the canonical multiplication map $R(G)_{N} \otimes R(H) \rightarrow R(G)$ as above. Hence $R(G)=R^{H}(G) \cdot R^{N}(G) \subset A(G)$, proving that $R(G)=A(G)$. Hence by Theorem $1, G$ has the property $(R)$ and our proof is complete.

Let $G$ be a connected reductive Lie group. Its Lie algebra $\mathfrak{G}$ is a direct sum $\mathfrak{G}=\mathfrak{Z} \oplus[\mathfrak{G}, \mathfrak{G}]$, where $\mathfrak{Z}$ is the center of $\mathfrak{G}$ and $[\mathfrak{G}, \mathfrak{G}]$ is the commutator ideal of $\mathfrak{G}$. Let $\mathfrak{G}$ (resp. $\mathfrak{K}_{1}$ ) be the direct sum of all noncompact (resp. compact) simple ideals of the semisimple Lie algebra $[\mathfrak{G}, \mathfrak{G}]$, and let $S$ (resp. 
$K_{1}$ ) be the analytical subgroup of $G$ corresponding to $\mathfrak{G}$ (resp. $\mathfrak{K}_{1}$ ). Thus $\mathfrak{G}=\mathfrak{Z} \oplus \mathfrak{K}_{1} \oplus \mathfrak{G}$, and if we put $K=Z K_{1}$, then $G=K \cdot S$, and $K \cap S$ is finite. We call $S$ (resp. $K$ ) the noncompact (resp. the compact) semisimple factor of $G$.

Now we are ready to characterize the connected reductive Lie groups having the property $(R)$.

Theorem 4. Let $G \subset \mathrm{GL}(n, \mathbb{R})$ be a connected reductive Lie group, and let $K$ (resp. $S$ ) be the compact (resp. noncompact) factor of $G$. Then $G$ has property (R) if and only if $S$ has property (R) and $K \cap S^{*} \subset S$.

Proof. Since $G$ is a connected reductive group, $G=K S$. Assume $G$ has the property $(\mathrm{R})$. Pick a representation $\rho$ of $G$ with $\operatorname{Ker}(\rho)=S$. This is possible since $G / S$ is faithfully representable as a compact group. Since $\rho$ is rational, $\rho$ maps $K \cap S^{*}$ to 1 and hence $K \cap S^{*} \subset S$ follows. To show that $S$ has the property $(\mathrm{R})$, it suffices to show that the inclusion $l: S \rightarrow S^{*}$ is the universal complexification of $S$ (Theorem 1). Let $\varphi: S \rightarrow \operatorname{GL}(m, \mathbb{C})$ be any representation of $S$ and view $\varphi$ as a representation of $S$ in an $m$ dimensional $\mathbb{C}$-linear space $V$. Since $G=K S$ and since $K$ is compact, there exists a representation $\varphi^{\prime}: G \rightarrow \mathrm{GL}\left(V^{\prime}, \mathbb{C}\right)$ so that $V$ is an $S$-subspace of the $G$-space $V^{\prime}$. Since $G$ has the property $(\mathrm{R}), \varphi^{\prime}$ extends to $\varphi^{\prime *}: G^{*} \rightarrow$ $\mathrm{GL}\left(V^{\prime}, \mathbb{C}\right)$. Since $\varphi^{\prime *}(S)$ leaves $V$ invariant, $\varphi^{\prime *}\left(S^{*}\right)$ leaves $V$ invariant. Define $\varphi^{*}: S^{*} \rightarrow \mathrm{GL}(V, \mathbb{C})$ by $\varphi^{*}(x)=\varphi^{*}(x) \mid V$ for $x \in S^{*}$. Then the complex representation $\varphi^{*}$ is the unique extension of $\varphi$.

Conversely assume that $S$ has the property (R) and that $K \cap S^{*} \subset S$. To show that $G$ has the property (R), it is enough to show that the inclusion $G \rightarrow G^{*}$ is the universal complexification. Note $K \cap S^{*} \subset S$ implies that $K^{*} \cap S^{*}=K \cap S$. Thus if $\varphi: G \rightarrow \mathrm{GL}(V, \mathbb{C})$ is any representation, then we extend $\varphi \mid K$ and $\varphi \mid S$ to rational representations $\varphi_{1}^{*}: K^{*} \rightarrow \mathrm{GL}(V, \mathbb{C})$ and $\varphi_{2}^{*}: S^{*} \rightarrow \mathrm{GL}(V, \mathbb{C})$, respectively. Define $\varphi^{*}: G^{*}=K^{*} \cdot S^{*} \rightarrow \mathrm{GL}(V, \mathbb{C})$ by $\varphi^{*} \mid K^{*}=\varphi_{1}^{*}$ and $\varphi^{*} \mid S^{*}=\varphi_{2}^{*}$. Since $K^{*} \cap S^{*}=K \cap S, \varphi^{*}$ is well defined and is the unique extension of $\varphi$.

The following is an example of a semisimple real algebraic group whose underlying Lie group admits a continuous but nonrational representation.

Example. Let $H=\operatorname{SL}(3, \mathbb{R})$, and let $G=\operatorname{Ad}(H)$ where Ad denotes the adjoint representation of $H$. Then $G$ is a real algebraic subgroup of $\operatorname{GL}(8, \mathbb{R})$, and $G$ is not well embedded [2, p. 329]. By Theorem 1, there exists a continuous representation of $G$, which is not rational. To construct such a representation, note that Ad is an injection. Let $\rho$ denote the representation, which is the composition $G \stackrel{\mathrm{Ad}^{-1}}{\longrightarrow} H=\operatorname{SL}(3, \mathbb{R}) \subset \mathrm{GL}(3, \mathbb{C})$. The representation $\rho$ is not rational. For otherwise, Ad: $H \rightarrow G$ would be an isomorphism of real algebraic groups, and this would imply that the complexification $\operatorname{SL}(3, \mathbb{C})$ of $H$ is isomorphic with the complexification $\operatorname{Ad}_{\mathbb{C}}(\operatorname{SL}(3, \mathbb{C}))$ of $G$, where $\operatorname{Ad}_{\mathbb{C}}$ denotes the adjoint representation of the complex group $\operatorname{SL}(3, \mathbb{C})$. Of course, the latter assertion is absurd. For example, the center of $\operatorname{SL}(3, \mathbb{C})$ is of order 3 while the center of $\operatorname{Ad}_{\mathbb{C}}(\operatorname{SL}(3, \mathbb{C}))$ is trivial. 


\section{REFERENCES}

1. C. Chevalley, Theory of Lie groups, Princeton Univ. Press, Princeton, NJ, 1946.

2. Harish-Chandra, Lie algebras and Tannaka duality theorem, Ann. of Math. (2) 51 (1950), 299-330.

3. G. Hochschild, The structure of Lie groups, Holden-Day, San Francisco, CA, 1965.

4. G. Hochschild and G. D. Mostow, Representations and representative functions of Lie groups, Ann. of Math. (2) 66 (1957), 495-542.

5. G. D. Mostow, Fully reducible subgroups of algebraic groups, Amer. J. Math. 78 (1956), 200-221.

6. M. Sugiura, Some remarks on duality theorems of Lie groups, Proc. Japan Acad., Ser. A Math. 43 (1967), 927-931.

7. __ The Tannaka duality theorem for semisimple Lie groups and the unitary tricks, Manifolds and Lie Groups (Notre Dame, IN, 1980), Progr. Math., vol. 14, Birkhäuser, Boston, MA, 1981.

Department of Mathematics and Statistics, Case Western Reserve University, CleveLAND, OHIO 44106 